\title{
Review on Diagnosis the Plant Disease at Early Stage
}

\author{
Nikita Butala $^{1}$, Prof. Mrs. Mehzabin Shaikh ${ }^{2}$, Sanjna Sharma ${ }^{3}$, Sneha Sukumaran ${ }^{4}$ \\ Student, Information Technology, JSPM's Rajarshi Shahu College of Engineering, Pune, India ${ }^{1,3,4}$ \\ Professor, Information Technology, JSPM's Rajarshi Shahu College of Engineering, Pune, India ${ }^{2}$
}

\begin{abstract}
Identifying plant diseases is the key to prevent the losses in the yield and quantity of the agricultural product. Monitoring of Health and plant disease detection is very critical for sustainable agriculture. It is very much difficult to monitor the disease of plants manually. It always requires tremendous amount of work, experts in the plant diseases, and also require the excessive amount of processing time. Hence, image processing is mainly used technique for detection of plant diseases and their preventions. Disease detection involves the steps like image acquisition, image preprocessing, feature extraction, image segmentation and classification. This paper discussed the methods which are used for the detection of plant diseases using their leaves images and their prevention at the early stage. This paper also discussed prevention techniques and cures against the diseases caused to the leaves.
\end{abstract}

Keywords: Image processing, Image Segmentation, Pest identification, Genetic algorithm.

\section{INTRODUCTION}

Today India ranks second in the farm output worldwide. Agriculture is even now the largest economic sector and plays a major role in social and economic development of India. Agriculture in India is the means of livelihood of almost two thirds of the working people in India. India has almost over 210 million acres of farm land. Jowar,wheat, groundnuts, pulses, sunflower, and cereals are the major crops. Apple, banana, spot, grapes, oranges are the most common fruits. Sugarcane, cotton, chilli are the major commercial crops .Computer Vision Systems (CVS) is a method developed for agricultural applications, namely, detection of weeds, sorting of fruits in fruit processing, classification of grains ,recognizing the food products in food processing, medicinal plant recognition, etc. In all these techniques, digital images are acquired as the main domain using digital camera and main is image processing techniques and are applied on these images which can help to extract the useful features required. In the past decade, agricultural applications using image processing and also the pattern recognition techniques have been attempted by many of the researchers. There is a need for design of a machine vision system that automatically helps in recognizing and classifying plant disease symptoms. Many diseases exhibit general symptoms which are caused by different pathogens produced by leaves, fruits, stem/stalk, roots, etc. Plant diseases are caused by bacteria, fungi, virus, nematodes, etc., of which fungi is mainly responsible for a large number of disease symptoms in plants.

India is a cultivated country and about $70 \%$ of the population of India depends on agriculture. Agriculture is the primary sector of India in which mostly all the population are dependent. Farmers have large range of diversity to select various suitable crops and to find the suitable pesticides for plants. Disease which is affectedon plants leads to the significant reduction in both the quality and quantity of agricultural products and is harmful for the living of any human beings. The study of plant disease is referred to the studies of visually what is being observed patterns on the plants. Monitoring of health and disease on plant is playing a very important role in the success of cultivation of crops in the farm. In early days, the monitoring and analysis of plant diseases were done manually by the expertise person in that field. This requires a large amount of work and also requires tremendous processing time. Image processing techniques can be used in the plant disease detection and it plays a major role. In many cases disease symptoms are seen on the leaves, stem, root and fruit. The plant leaf for the detection of disease is considered which shows the disease symptoms.

India is a cultivated country and about $70 \%$ of the population depends on agriculture. Farmers have large range of diversity for selecting various suitable crops and finding the suitable pesticides for plant. Disease on plant leads to the significant reduction in both the quality and quantity of agricultural products. The studies of plant disease refer to the studies of visually observable patterns on the plants. Monitoring of health and disease on plant plays an important role in successful cultivation of crops in the farm. In early days, the monitoring and analysis of plant diseases were done manually by the expertise person in that field. This requires tremendous amount of work and also requires excessive processing time. The image processing techniques can be used in the plant disease detection. In most of the cases disease symptoms are seen on the leaves, stem and fruit. The plant leaf for the detection of disease is considered which shows the disease 
symptoms. This paper gives the introduction to image processing technique used for plant disease detection.

Plant disease identification when considered through visual manner is a laboratory task and at the same time less accurate and can be done only in limited areas. Whereas if automatic detection technique is used it will take less efforts, less time and more accurately. In plants, some general diseases are brown and yellow spots, or early and late scorch, and other are fungal, viral and bacterial diseases. Image processing is the technique which is used for measuring affected area of disease, and to determine the difference in the color of the affected area.

Image segmentation is the process of separating or grouping an image into different parts. There are currently many different ways of performing image segmentation, ranging from the simple thresholding method to advanced colour image segmentation methods. These parts normally correspond to something that humans can easily separate and view as individual objects. Computers have no means of intelligently recognizing objects, and so many different methods have been developed in order to segment images. The segmentation process in based on variousfeatures found in the image. This might be color information, boundaries or segment of an image. Genetic algorithmsbelong to the evolutionary algorithms which generate solutions for optimization problems. Algorithm begins with a set of solutions called population. Solutions from one population are chosen and then used to form a new population. This is done with the anticipation, thatthe new population will be enhanced than the old one. Solutions which are selected to form new solutions (offspring) are chosen according to their fitness - themore appropriate they are the more probability they have to reproduce masking is done for the useful segments that are extracted first in this step, while image is segmented. And in last or fourth main step the segmentation is done approach given in this for feature set extraction is the Color Co-occurrence Method. For automatic detection of diseases in leaves, neural networks are used. The approach proposed can significantly support an accurate detection of leaf, and seems to be important approach, in case of steam, and root diseases, putting fewer efforts in computation.

\section{II.LITERATURE REVIEW}

According to Paper [1] disease identification process include some steps out of which four main steps are as follows: First input is RGB image, then a colour transformation structure is taken, and afterwards using a specific threshold value, the green pixels are masked and removed, then further it is followed by segmentation process, and to get useful segments the statistics of structure is computed. At last, classifier is used for the features that are extracted to classify the disease. The proposed algorithm shows its efficiency with an accuracy of $94 \%$ in successful detection and classification of the examined diseases.
According paper [2] in this paper detection of diseases on leaf has been used method is twofold: 1) identifying the affected object based on k-means clustering algorithm;2) taking the features set of the affected or infected objects for colour texture analysis we will use colour cooccurrence methodology.

In paper [3] there are four main steps in processing developed scheme, in which, one is for the RGB input image, a colour structured transformation is created, because for the colour generation RGB is used and converted image or transformed of RGB, that is the, HSI is used as the colour descriptor. In the second step of this, by using the threshold value, the green pixels are masked and removed. In third, by using precompiled level threshold, green pixels and masking removing is done for the useful segments that are taken in first step. In this step, as the image is measured or segmented. In last step or in fourth main step the segmentation is done.

According to Paper [4] identification of diseases process includes some steps in which the four main steps are as given below: first, for the input RGB image, a colour transformation structure is inputted, and then using a specified threshold value, the removal and masking of the green pixels is done, which is followed by process segmentation, and for getting useful segments the statistics and texture are computed. Finally, the classifier is used for the extraction of the features to classify the diseases. The proposed algorithm shows its accuracy and the efficiency and the accuracy of $94 \%$ in successful identification and classification of the diseases detection.

According to the Paper [5] which presents disease detecting process in domestic through an expected method like colour analysis and texture, K-mean clustering. To identify, classify and recognize different agriculture, it uses the colour features and texture those generally appear in normal and infected areas. In future, for classifying purposed and given classifiers can also be use, such as Bayes classifier, principal component classifier and $\mathrm{K}$ means clustering.

According to Paper [6] represents the Triangles threshold and methods of simple thresholds. These techniques are used to lesion region area and segmentation of the leaf area respectively. In last step, categories of disease are done by calculating the leaf area and lesion area. According to the research done yet, the given method is efficient, fast and has accuracy for the calculation of leaf disease severity and leaf area calculation is done by using threshold segmentation.

\section{BASIC STEPS FOR DISEASE DETECTION}

According to this session, the basic steps of the plant disease identification and classification is done using image processing which are shown (Fig. 1)).Basic steps for plant disease detection are as follows: 


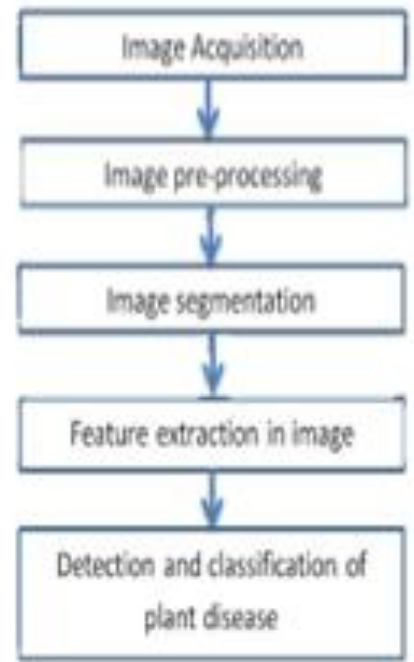

\section{BASIC STEPS FOR DISEAES DETECTION}

\section{A] Image Acquisition}

The image of the plant leaf is clicked through the camera device. This image is in the form of RGB (Red, Green and Blue). Colour transformation structure for the RGB leaf image is created, and a device-independent colour space transformation for the colour transformation structure.

\section{B] Image pre-processing}

Image pre-processing is the process to perform some operation on image in order to enhance the image or to extract some useful information about from it. It is type of signal processing in which inputted image or associated feature with that image. It can forms core research area within engineering and computer science disciplined.

\section{C] Image Segmentation}

Segmentation is nothing but partitioning of image into various parts of same features or having some similarity. Image segmentation is the process of partitioning a digital image in to multiple segments such as sets of pixels which is also called as super-pixels.

The main goal of segmentation is to simplify and change the representation of an image into something which is more meaningful and easier to analyse. Image segmentation is typically used to locate objects and boundaries like lines, curves, etc. in images The segmentation can be done using various methods like kmeans clustering, converting RGB image into HIS model etc.

\section{D] Feature Extraction}

Feature extraction plays an important role for identification of an various object. In many application of image processing feature extraction is used.

Color, texture, edges, morphology etc. are the features which can be used in plant disease detection. Feature extraction is related to reduction in dimension. When the input date to an algorithm is too large to be processed and suspected to redundant in both feet and meters, or the receptiveness of images presented as pixel then it can be transformed into a reduced set of features, this is called as feature selection.

E]Detection and Classification of plant disease

Classification of remotely sensed data is used to assigned corresponding levels with respect to groups with homogeneous characteristics, with the aim of discriminating multiple objects from each other within the image.

These level is called class. Classification will be executed on the base of spectral or spectrally defined features, such as density, texture etc. in the feature space. It can be said that classification divides the feature space into several number of classes based on a decision algorithm.

Step 1: Definition of Classification Classes

Depending on the objectives and the characteristics of the image data,classes of classification should be clearly defined.

\section{Step 2: Selection of Features}

Features to discriminate between the classes should be established using multi-spectral and/or multi-temporal characteristics, textures .color,etc.

\section{Step 3: Sampling of Training Data}

Training data should be sample in order to determined appropriate decision rules.Various classification techniques such as supervised or unsupervised learning will then be selected on the basis of the training data sets.

Step 4: Estimation of the Universal Statistics

Various classification techniques will be compared with the training data, so that an appropriate decision rules are selected for subsequent classification.

\section{Step 5: Classification}

Depending up on the decision rules, all pixels are classified in a single class. There are two methods of pixel by pixel classification and per-field classification, with respect to segmented areas.

Popular techniques are as follows:

a. Multi-level slice classifiers

b. Minimum distance classifiers

c. Maximum likelihood classifiers

d. Other classifiers such as fuzzy set theory and expert systems

Step 6: Verification of Result

The classified results should be checked and verified for their accuracy and reliability. 

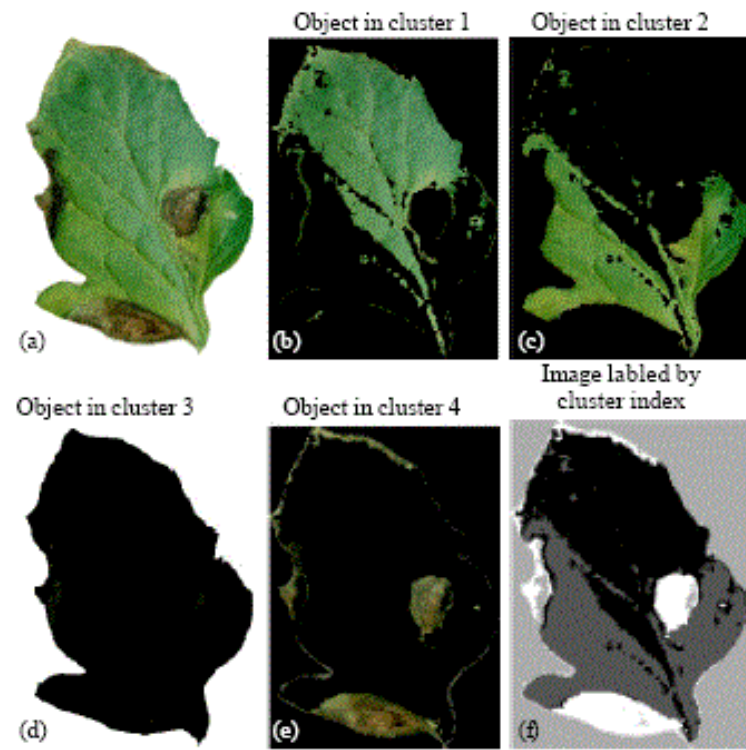

Image labled by cluster index

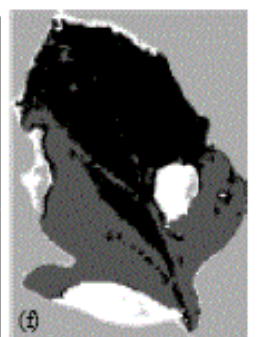

\section{PROPOSED SYSTEM}

We can detect insects of multiple colours and now many algorithms are implemented for the identification of different colours. This system is being developed for the detection of the diseases of plants such as flowering and non- flowering plants . This is a desktop based application and it is cost efficient. There is no language barrier in this software system. Languages provided are Hindi and English.

The objectives of this proposed system are as follows: 1. To make an efficient use of image processing techniques.

2. Provide solution with least hardware requirement.

3. To develop a Desktop application that is cost efficient.

4. Minimize the use of resources.

5. Easy to use and accurate.

\section{CONCLUSION}

In this technique we have learned the Plant Disease Detection which describes different methods of plant disease and it helps us to know and understand Plant Disease at the early stage itself. The Feature Extraction, Color Co-Occurrence Matrix, Color Imaginary Transform will be done and then we will get a desired result. Image Processing-Based approach is proposed and it is useful for Plant disease detection. This system then describes different Techniques of Image Processing for several Plant species which are been used for detecting the plant disease. The Disease of the plant is known at an early stage and the cure is suggested using different languages. The Image Processing Techniques applied for identification and classification of fungal disease affected on different Agriculture/Horticulture crops are summarized.

\section{REFERENCES}

[1] Ms.Kiran R. GavhaleDept.of Computer Technology research paper of international conference technology

[2] 2014-15

[3] Haiguang Wang, Guanlin Li, Zhanhong Ma, Xiaolong Li, "Image Recognition of Plant Diseases Based on Back propagation Networks, 5th International Congress on Image and Signal Processing, pp-894900,Chongqing, China, 2014

[4] A.Menukaewjinda, P.Kumsawat, K.Attakitmongcol, A.Srikaew, "Grape leaf disease detection from color imagery using hybrid intelligent system", Proceedings of electrical Engineering/electronics,

[5] Telecommunications and Information technology (ECTICON), vol

[6] S. Arivazhagan , R. NewlinShebiah , S. Ananthi, S. Vishnu classification of plant leaf diseases using texture features", Commission Internationale du Genie Rural(CIGR) journal, vol. 15, no.1, pp:211-217, March 2013

[7] Song Kai, liuzhikun, Su hang, Guochunhong, "A Research of maize disease image recognition of Corn Based on BP Networks", Third International Conference on Measuring Technology and Mechatronics Automation,pp:246-249, Shenyang, China, 2011.

[8] H. Al-Hiary, S. Bani-Ahmad, M. Reyalat, M. Braik and Z. ALRahamneh, "Fast and Accurate Detection and Classification of Plant Diseases", International Journal of Computer Applications, Wageningen Academic publishers, vol. 17, no.1, pp: 31-38, March 2011.

[9] Yan-Cheng Zhang, Han-Ping Mao and Bo Hu, Ming-Xi Li, "Features selection of cotton diseases leaves images based On fuzzy features selection techniques", International Conference on Wavelet Analysis and Pattern Recognition, Beijing, pp. 124-129, 2007

[10] ShenWeizheng, Wu, Yachun, Chen Zhanliang and Wei Hongda, "Grading method of leaf spot disease based on image processing", Proceedings Of 2008 International Conference On Computer Science And Software Engineering, Volume 06,2008.

[11] J. A. Tsanakas, D. Chrysostomou, P. N. Botsaris, and A. Gasteratos, "Fault diagnosis of photovoltaic modules through image processing and Canny edge detection on field thermographic measurements," International Journal of Sustainable Energy, vol. 34, no. 6, pp. 351-372, Jul. 2015.

[12] G. Leotta, P. M. Pugliatti, A. D. Stefano, F. Aleo, and F. Bizzarri, "Post processing technique for thermo-graphic images provided by drone inspections," in Proceedings of the 31st European Photovoltaic Solar Energy Conference and Exhibition (EU PVSEC 2015), 2015, 